\title{
The Terahertz Controlled Duplex Isolator: Physical Grounds and Numerical Experiment
}

\author{
Konstantin Vytovtov, ${ }^{1}$ Said Zouhdi, ${ }^{2}$ Rostislav Dubrovka, ${ }^{3}$ and Volodymyr Hnatushenko \\ ${ }^{1}$ Department of Physics, Electronics and Computing Systems, Oles Honchar Dnipropetrovsk National University, \\ Gagarina 72, Dnipropetrovsk 49010, Ukraine \\ ${ }^{2}$ Laboratoire de Génie Electrique de Paris, SUPELEC, 91192 Gif-sur-Yvette, France \\ ${ }^{3}$ Queen Mary University of London, UK
}

Correspondence should be addressed to Volodymyr Hnatushenko; vvgnatush@gmail.com

Received 27 June 2015; Revised 23 November 2015; Accepted 24 December 2015

Academic Editor: Dmitry Kholodnyak

Copyright (C) 2016 Konstantin Vytovtov et al. This is an open access article distributed under the Creative Commons Attribution License, which permits unrestricted use, distribution, and reproduction in any medium, provided the original work is properly cited.

Electromagnetic properties of an anisotropic stratified slab with an arbitrary orientation of the anisotropy axis under an oblique incidence of a plane harmonic wave are studied. The dependence of the eigenwave wavenumbers and the reflection coefficient on an anisotropy axis orientation and frequency is investigated. For the first time, the expression for the translation matrix is obtained in the compact analytical form. The controlled two-way dual-frequency (duplex) isolator based on the above described slab is presented for the first time. It is based on the properties of the anisotropic structure described here but not on the Faraday effect.

\section{Introduction}

The terahertz domain actively developed in the last decades [1-5]. Various devices have been created and numerous electromagnetic structures have been used in this frequency range. One of the most interesting classes of terahertz devices is the class of nonreciprocal devices [1-10]. Usually, the Faraday rotation effect itself is exploited naturally to achieve desired nonreciprocal performance [1]. The electrically tunable Faraday effect in a $\mathrm{HgTe}$ thin film has been reported in [2]. Faraday rotation has also been studied for pump pulse of terahertz radiation [3]. In [4], the heterostructures based on the piezoelectric and semiconductor layers have been proposed for radio frequency applications. Other examples of nonreciprocal properties of nonlinear devices in terahertz range have been presented in [5] where a Faraday isolator operating on the coupled microwave resonators circuit has been proposed. A theoretical study of the reflection of infrared radiation from antiferromagnets and $M$-type barium hexagonal ferrite using an attenuated total reflection geometry has been presented in [6]. Nonreciprocal devices using attenuated total reflection and thin film magnetic layered structures have been described in [7]. Thus, we can clearly see that this topic is quite relevant in modern microwave science.

In this paper, we consider nonreciprocal properties of an anisotropic structure based on dependence of the reflection coefficient on an incident wave direction and an anisotropy axis orientation, but not on the Faraday effect. The physical grounds and numerical simulations of a controlled bidirectional dual-frequency isolator not based on the Faraday effect are presented. For this, the analytical investigation and numerical calculations of a stratified anisotropic structure are carried out. At first, the elements of the translation matrix are written in the compact analytical form (4) for the general case of an anisotropic medium for the first time. It is important that these elements are expressed in elementary algebraic functions. The obtained form is very useful in subsequent numerical experiments.

In this work, it also is found that the reflection coefficients are different for different incidence angles $\alpha$ and $-\alpha$. Thus, a structure shows nonreciprocal properties.

These properties allow us to build nonreciprocal elements in the terahertz domain. The physical principles of construction of a two-way double-frequency (duplex) isolator are described here. These principles are based on dependence 


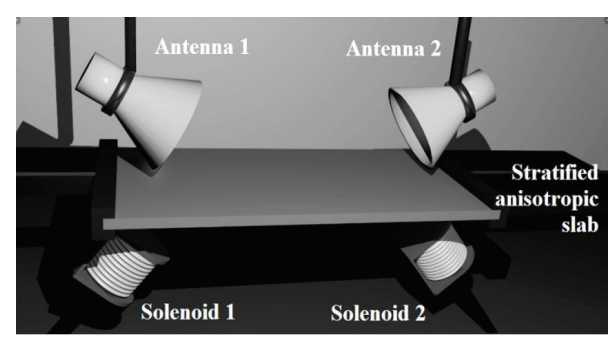

(a)

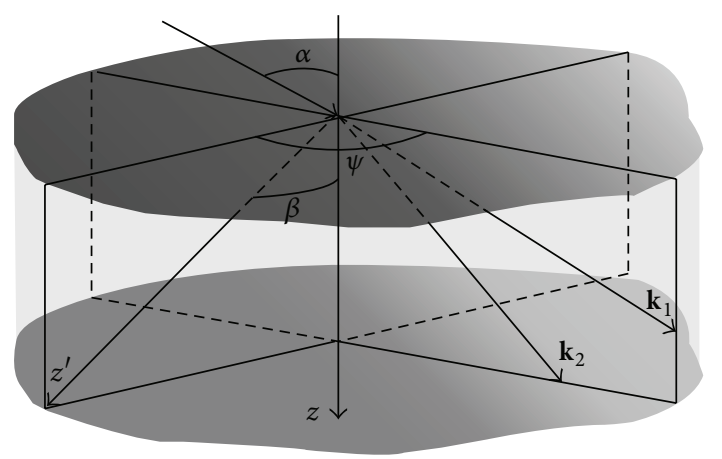

(b)

Figure 1: (a) Appearance of the controlled double-frequency bidirectional isolator; (b) geometry of an anisotropy layer.

of reflection coefficient on an incidence wave direction and an orientation of an anisotropy axis. It should be noted that the Faraday effect is also present in these structures and also defines nonreciprocal properties of anisotropic structures. Today, a wide variety of isolators is described in contemporary scientific literature [7-9]. A feature of the presented device is the fact that it is a two-way dualfrequency one. Switching an isolator (changing a direction and a frequency) is implemented by two solenoids. Its tuning is done mechanically by changing an orientation of external magnetic field. Note that here a very complicated geometry of the problem is used to create the isolator. But only this geometry allows us to obtain the pronounced minimums of reflectance for a given incidence angle, as well as a wide range of total reflection.

\section{Description of the Isolator Scheme}

A presentation of the offered duplex isolator is shown in Figure 1(a). This device contains two transceiver antennas, two solenoids generating a magnetizing field, and a stratified anisotropic slab (a nonreciprocal element).

An orientation of the axes within a homogeneous layer of a stratified anisotropic slab is presented in Figure 1(b). Here, $z^{\prime}$ is an anisotropy axis, $z$ is a normal to a structure, $\mathbf{k}_{1}$ and $\mathbf{k}_{2}$ are wave vectors of forward refracted waves within an anisotropic layer, $\beta$ is an inclination angle, $\psi$ is an angle between an incidence plane and a plane containing an anisotropy axis, and $\alpha$ is an incidence angle. The choice of such a complicated geometry is due to the need to obtain an asymmetric (nonreciprocal) structure, the need to obtain wide range of total reflection, and the need to control the structure. Of course nonreciprocal properties can be observed in the simplest case of orientation of an anisotropy axis along interfaces $[10,11]$, but in that case total reflection regions are not sufficiently wide and a structure has low selective properties in comparison with the given case. Additionally, here mechanical tuning of an isolator is assumed. And using such a complicate geometry gives us the additional freedom to control the device. Moreover, properties of a structure having such a geometry are poorly understood. Therefore, the results of these investigations have both practical and theoretical significance.

The layers of a stratified structure are described by a scalar permittivity and a dyadic permeability in the gyrotropic form:

$$
\mu=\left|\begin{array}{ccc}
\mu_{x x} & j \mu_{x y} & 0 \\
-j \mu_{x y} & \mu_{x x} & 0 \\
0 & 0 & \mu_{z z}
\end{array}\right|
$$

with

$$
\begin{aligned}
& \mu_{x x}=1+\frac{\omega_{M}\left(\omega_{0}+j / T\right)}{\left(\omega_{0}+j / T\right)^{2}-\omega^{2}}, \\
& \mu_{x y}=\frac{\omega_{M} \omega}{\left(\omega_{0}+j / T\right)^{2}-\omega^{2}} .
\end{aligned}
$$

Here, $\omega_{M}=4 \pi \gamma M, \omega_{0}=\gamma H_{0}, \gamma$ is the gyromagnetic relation, $H_{0}=0.1 T$ is an external magnetic field, and $T$ is a relaxation time.

In our numerical investigation, we take into account losses and frequency dispersion of the ferrite material and the elements in (1) are written in the Lanfau-Lifshits form [12]. The dependence of the permeability elements on a frequency for the first and second layers of a period is given in Figure 2. Here, $\mu_{x x}^{\prime}$ and $\mu_{x y}^{\prime}$ are the real parts of the elements, and $\mu_{x x}^{\prime \prime}$ and $\mu_{x y}^{\prime \prime}$ are the imaginary parts of the elements. The resonance frequencies are $1.1 \mathrm{GHz}$ and $1.8 \mathrm{GHz}$ for the first and second layers accordingly.

\section{Method of Calculation}

The well-known translation matrix method and the reflection matrix method $[13,14]$ are used in this study of a stratified anisotropic slab. Both of these methods are accurate and analytical and these can be used in any frequency range for both lossless and lossy media without restrictions. According to the definition, a translation matrix relates tangential field components on both boundaries of a plane-parallel structure:

$$
\left|\begin{array}{l}
E_{x}(z) \\
E_{y}(z) \\
H_{x}(z) \\
H_{y}(z)
\end{array}\right|=\mathbf{M}(z)\left|\begin{array}{l}
E_{x}(0) \\
E_{y}(0) \\
H_{x}(0) \\
H_{y}(0)
\end{array}\right| .
$$

Here, $\mathbf{M}(z)$ is a $2 \times 2$ matrix for an isotropic medium or a $4 \times 4$ translation matrix for an anisotropic medium.

The method is described in the literature in detail for both isotropic [13] and anisotropic [14] media. The matrices have been found for a normal orientation of an anisotropy axis, 


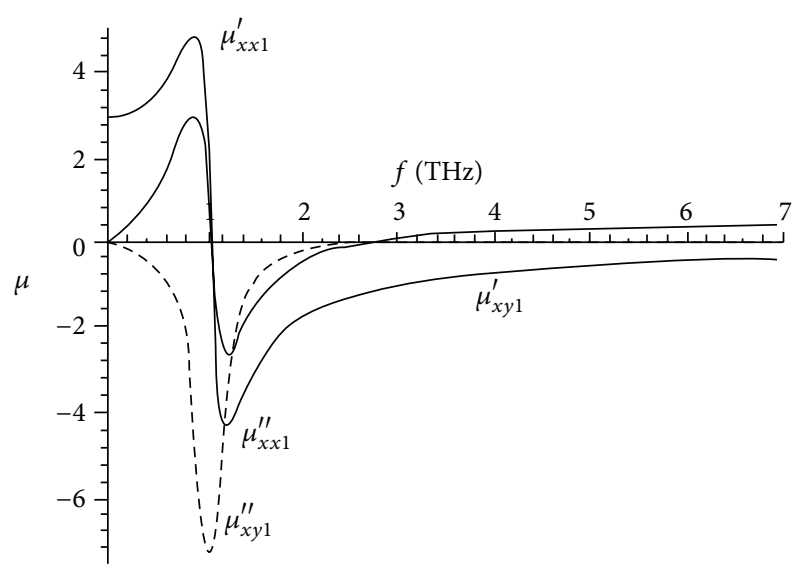

(a)

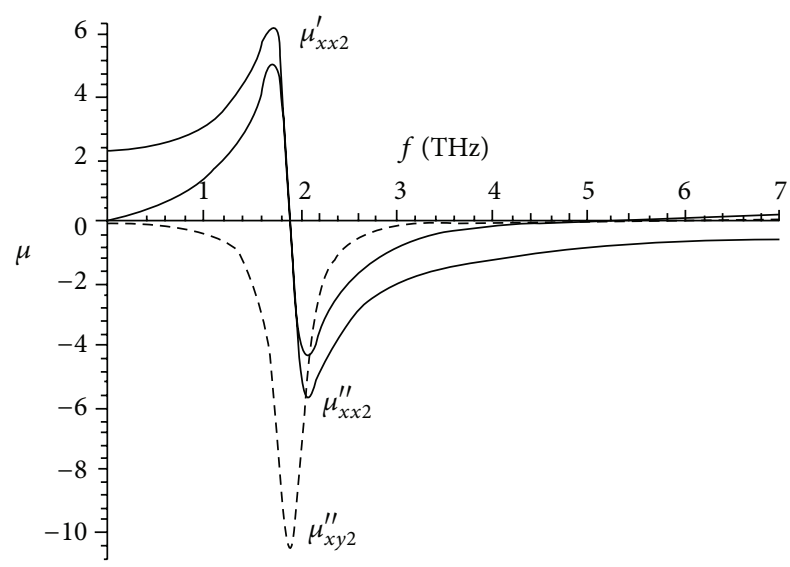

(b)

FIgURE 2: The dependence of permeability elements on frequency for the first (a) and second (b) layers of a period.

for a tangential orientation of an anisotropy axis, for arbitrary orientation of an anisotropy axis, and for a normal incidence of a plane harmonic wave. However, the expressions for the most general case of an anisotropic medium with an arbitrary orientation of the anisotropy axes under an oblique incidence of a wave have not been obtained in a compact analytical form. In this paper, the translation matrix elements of a homogeneous layer are presented for the first time as

$$
M_{m l}=\sum_{i=1}^{4}(-1)^{m+i} \gamma_{m i} \frac{\operatorname{det} \mathbf{G}_{l i}}{\operatorname{det} \mathbf{G}} \exp \left(-j k_{i} z\right) .
$$

Here, $\mathbf{G}_{l i}$ is the minor of the element $\gamma_{m i}$ of the $4 \times 4$-matrix G. Matrix $\mathbf{G}$ is defined by the expression

$$
\begin{aligned}
\left|\begin{array}{l}
E_{x} \\
E_{y} \\
H_{x} \\
H_{y}
\end{array}\right| & =\left[\begin{array}{llll}
\gamma_{11} & \gamma_{12} & \gamma_{13} & \gamma_{14} \\
\gamma_{21} & \gamma_{22} & \gamma_{23} & \gamma_{24} \\
\gamma_{31} & \gamma_{32} & \gamma_{33} & \gamma_{34} \\
\gamma_{41} & \gamma_{42} & \gamma_{43} & \gamma_{44}
\end{array} \mid\left[\begin{array}{l}
A_{1} \exp \left(-j k_{1} z\right) \\
A_{2} \exp \left(-j k_{2} z\right) \\
A_{3} \exp \left(-j k_{3} z\right) \\
A_{4} \exp \left(-j k_{4} z\right)
\end{array}\right]\right. \\
& =\mathbf{G}\left[\begin{array}{c}
A_{1} \exp \left(-j k_{1} z\right) \\
A_{2} \exp \left(-j k_{2} z\right) \\
A_{3} \exp \left(-j k_{3} z\right) \\
A_{4} \exp \left(-j k_{4} z\right)
\end{array}\right],
\end{aligned}
$$

where $A_{i}$ is a constant. Here also $k_{i}$ is a wavenumber defined by well-known fourth-order dispersion relation [12-16]:

$$
k^{4}+a_{3} k^{3}+a_{2} k^{2}+a_{1} k=0 .
$$

The coefficients $a_{i}$ in (6) must be obtained directly from Maxwell equations.

The expression of matrix $\mathbf{G}$ in (5) can be obtained directly from Maxwell equations. Actually, expression (5) is a general solution of Maxwell's equations for this case [12-14] (see Appendix). The achieved form of the translation matrix (4) essentially simplifies the solution of the programming problem and improves accuracy of numerical calculations. Moreover, no analytical expression of the translation matrix element has been presented in scientific literature for this complicate case.

The reflection matrix relates the tangential components of incident and reflected waves [15]:

$$
\left|\begin{array}{l}
E_{x \text { refl }} \\
E_{y \text { refl }}
\end{array}\right|=\mathbf{R}\left|\begin{array}{l}
E_{x \text { inc }} \\
E_{y \text { inc }}
\end{array}\right| .
$$

Here $E_{x \text { inc }}$ and $E_{y \text { inc }}$ are the components of an incident field, $E_{x \text { refl }}$ and $E_{y \text { refl }}$ are the components of a reflected field, and $\mathbf{R}$ is a $2 \times 2$ reflection matrix.

\section{Nonreciprocal Properties of Anisotropic Structure}

The main element of the isolator is a stratified anisotropic slab (Figure 1) possessing nonreciprocal properties. In this section, we consider the main physical features of a slab, which ensure its isolator properties. An orientation of the axes for the studied case is shown in Figure 1(b). Angle $\psi$ is given in Figure 1(b). The presented complicated geometry of the problem gives us the interesting features of electromagnetic field in the structure. Such properties cannot appear in a case of more simple geometry. In particular, study of wavenumbers in a homogeneous anisotropic layer suggests that the wavenumbers can be real, imaginary, or complex even in a lossless medium. In the considered case, imaginary parts of wavenumbers indicate existence of a complex wave [16] but not in presence of losses in an anisotropic structure. Indeed a resulting structure of electromagnetic field in a layer is determined by a superposition of all four eigenwaves only, but not each eigenwave separately. In addition, these numbers are not the same for the incidence angles $\alpha$ and $-\alpha$ (Figure 3 ). The numerical calculations analytical investigations give us the following result (Figure 4): $k_{1}(\alpha)=-k_{4}(-\alpha), k_{2}(\alpha)=$ $-k_{3}(-\alpha), k_{3}(\alpha)=-k_{2}(-\alpha)$, and $k_{4}(\alpha)=-k_{1}(-\alpha)$. Thus, we can see nonreciprocal properties of the medium in the considered case.

It also is clear (Figure 3) that two of the four wavenumbers can be real and two others can be complex for the certain parameters of the structure $\left(10^{\circ}<\alpha<16^{\circ}\right)$. It should also be noted that all four wave numbers have positive (negative) real part (Figure 3 ) in the certain range of the incidence angles $\left(\alpha>16^{\circ}\right)$. Such unusual combinations of wavenumbers are possible only in the case of this complex geometry 


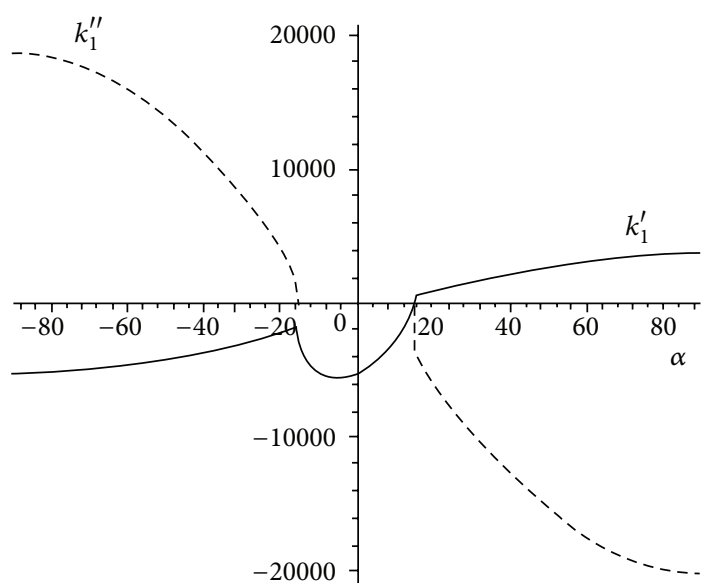

(a)

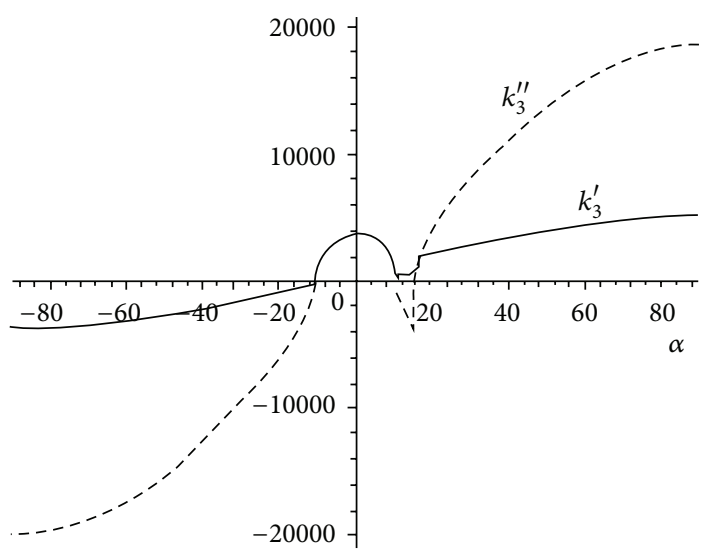

(c)

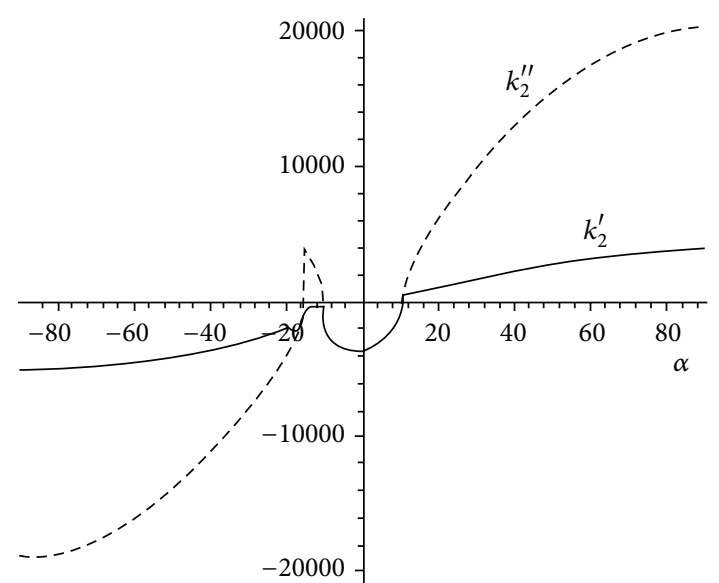

(b)

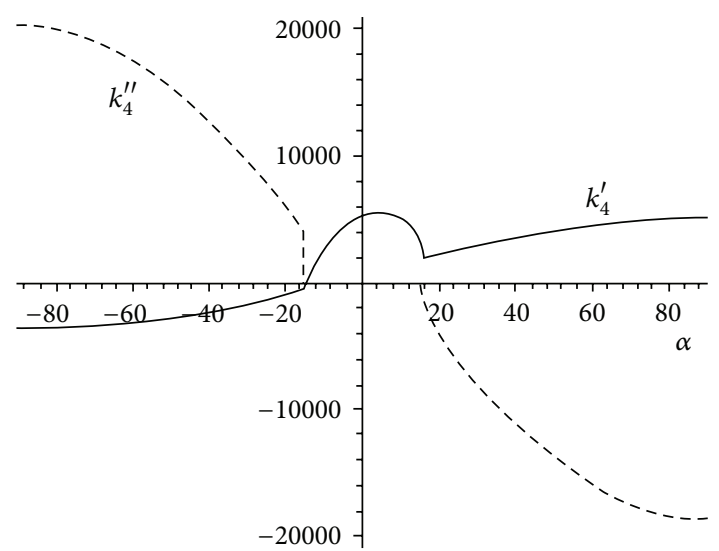

(d)

FIGURE 3: The dependence of the wavenumbers on an incidence angle $\left(\beta=60^{\circ}, \psi=30^{\circ}, f=10 \mathrm{THz}, \mu_{x x}=4.9, \mu_{x y}=2.9\right.$, and $\left.\mu_{z z}=0.99\right)$.

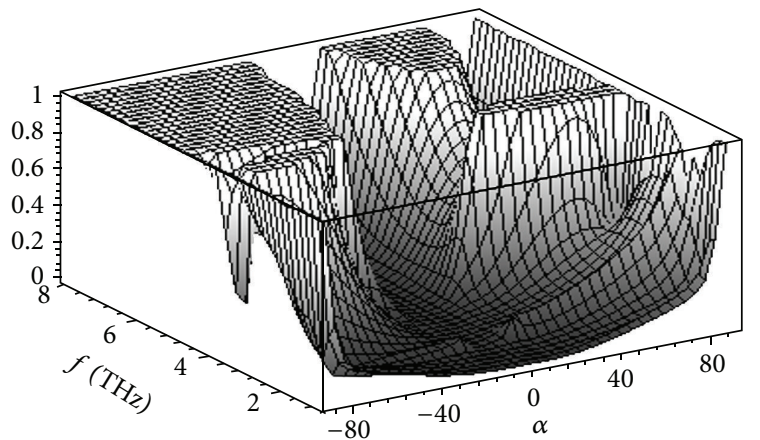

FIGURE 4: The dependence of the reflection on a frequency and an incidence angle $\left(\beta=30^{\circ}, \psi=50^{\circ}\right), d_{1}=0.78 \mathrm{~mm}$, and $d_{1} / d_{2}=1.3125$.

of the problem. Similarly, it also is verified that the field components are different for the incidence angles $\alpha$ and $-\alpha$.

Obviously, this kind of wavenumber affects reflection properties of a medium. So now we analyze the dependence of the reflection coefficient on a frequency and an anisotropy axis orientation. One of the possible variants of this dependence is presented in Figure 4. The study shows that there is no regularity in the reflection coefficient dependence on a frequency and an orientation of the anisotropy axis. Here a very wide range of total reflection even for small incidence angles can be seen. Their existence is determined by multiple reflection phenomenon, existence of four eigenwaves with different wavenumbers and propagation angles in each layer, presence of complex waves, and the losses in the medium. The resonant character of the reflection coefficient is determined by the effects described above also. Note that there are no sharp edges of the reflection coefficient in the neighborhood of the unity. It also is shown (Figure 5) that the dependence of the reflection coefficient of a frequency is not symmetric with respect to $\alpha=0$. This is due to the asymmetric dependence of wave numbers and the field components on the incidence angle.

\section{Operation Principle of the Isolator}

The operation principle of the device is based on an angle dependence of medium properties (in particular, the reflection coefficient) but not on the Faraday effect. Wherein for the certain structure parameters and an orientation of the anisotropy axis (an external magnetic field direction) it is possible to obtain the total reflection at an incidence angle 


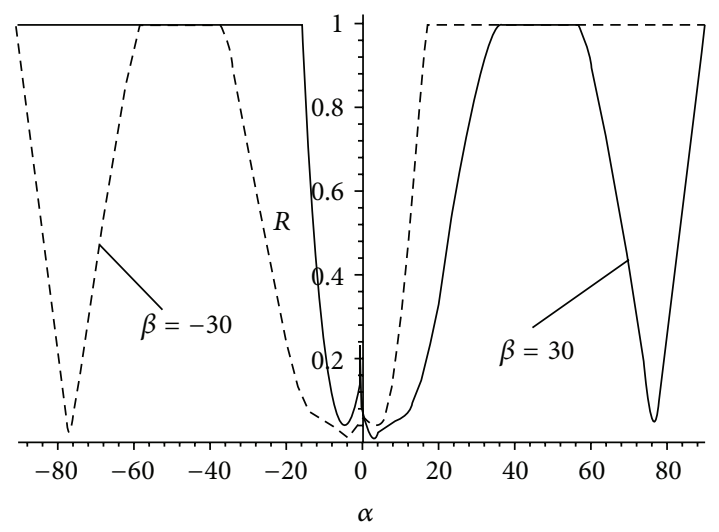

FIGURE 5: The dependence of the reflection coefficient on the incidence angle for $f=6.35 \cdot 10^{12}, \psi=50^{\circ}, d_{1}=0.78 \mathrm{~mm}$, and $d_{1} / d_{2}=1.3125$; (1) $\beta=30^{\circ}$ solid line and (2) $\beta=-30^{\circ}$ dash line.

$\alpha$ and the minimal reflection at an incidence angle $-\alpha$. Analogously, it is possible to choose an orientation of the external magnetic field for the given structure parameters such that it is possible to obtain the total reflection for $-\alpha$ and the minimal reflection for $\alpha$. Thus, changing a direction of wave propagation $(\alpha$ or $-\alpha)$ can be accomplished by changing a direction of an external magnetic field. Analogously it is possible to find the two frequencies that give such an effect.

Now let us describe, for clarity, the operation principle of a bidirectional double-frequency isolator shown in Figure 1. If a voltage is applied to solenoid 1 , a signal transmits from antenna 1 to antenna 2 without attenuation at a frequency $f_{1}$ as the reflection coefficient is equal to unite, a signal cannot propagate from antenna 2 to antenna 1 at this frequency as the reflection coefficient is about zero, and a signal transmits from antenna 2 to antenna 1 without attenuation at a frequency $f_{2}$; however, inverse propagation is not possible at this frequency. When voltage is applied to solenoid 2 , a signal transmits from antenna 2 to antenna 1 substantially without attenuation at a frequency $f_{1}$, and it transmits from antenna 1 to antenna 2 without attenuation at a frequency $f_{2}$. Circular aperture of the antennas is used because the Faraday effect (rotation of a polarization plane) is taken into account.

\section{The Parameters of the Isolator}

A very important characteristic of an isolator is its amplitude response. During the researches, numerous calculations have been carried out and the optimal structure for isolator is chosen. This structure includes the 12 double-layered periods. The first layer of the structure is $\mathrm{FeF}_{2}$ and the second one is $\mathrm{MnO}$. The antiferromagnetic resonance frequencies in the considered case are $1.1 \mathrm{THz}$ and $1.8 \mathrm{THz}$ for the first and second layers accordingly. The dependence of the element on a frequency is given in Figure 2. Here the operating frequency $f=6.35 \mathrm{THz}$ and the angles $\beta= \pm 30^{\circ}$ and $\psi=50^{\circ}$ are also taken. In our problem, the operating frequency is essentially higher than the resonant one. The losses in $\mathrm{FeF}_{2}$ and $\mathrm{MnO}$ can be neglected at this frequency (Figure 2). Here we consider an above resonance frequency and we have $\mu_{x x}=1.415$ and $\mu_{x x}$

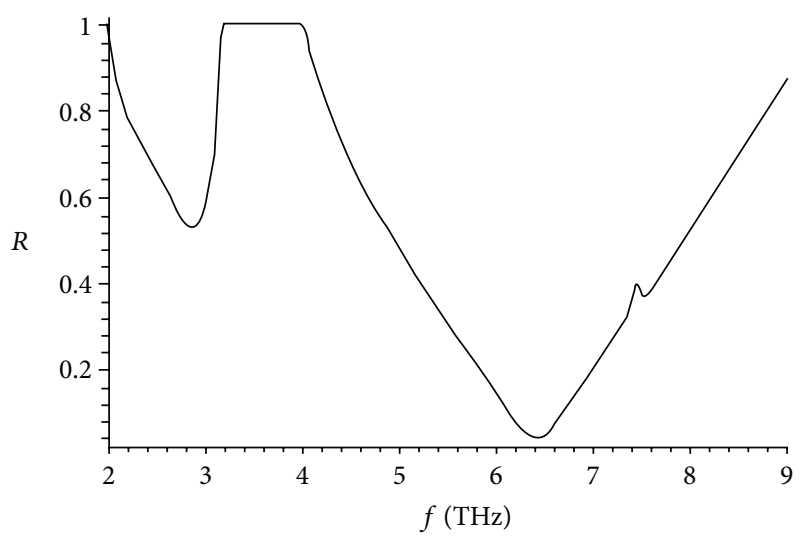

FIGURE 6: The dependence of reflection coefficient on a frequency at $\alpha=76.4^{\circ}, d_{1}=0.78 \mathrm{~mm}$, and $d_{1} / d_{2}=1.3125$.

$=-0.42$ for the first layer of the period at this frequency and $\mu_{x x}=1.2$ and $\mu_{x x}=-0.63$ for the second layer. In the given work we consider a TE incident wave. The dependence of the reflection coefficient on an incidence angle for this structure (the amplitude characteristic) is presented in Figure 5. It is seen that the reflection coefficient is minimal $(R=0.05)$ at $\alpha=76.4^{\circ}$ and the one is equal to unit at $\alpha=-76.4^{\circ}$ for $\beta=30^{\circ}$ (the solid line).

Thus, this structure has isolator properties and it passes a signal only in a forward direction and does not pass a signal in an opposite direction. The stopband for an opposite direction is $9.6^{\circ}$ at the level $0.707,15.8$ at the level 0.5 , and 27.7 at the level 0.1. Now, let us chose $\beta=-30^{\circ}$ (the dash line). For this case, the minimum of the reflection coefficient $(R=0.03)$ is at $\alpha=-76.4^{\circ}$.

The frequency dependence of the reflection coefficient is presented in Figure 6. This graph is presented for the incidence angle $\alpha=76.4^{\circ}$. It is obvious that this structure has bad frequency selective properties and low squareness coefficient. The bandwidth is $1.88 \mathrm{THz}(29.38 \%)$ at the 0.707 level (a half power level), and the bandwidth is $3.13 \mathrm{THz}$ $(48.9 \%)$ at the 0.5 level of the maximum. The squareness is 1.66 at levels 0.5 and 0.707 . It also is verified that changing orientation of magnetic field allows us to shift a reflection minimum in a small range. And changing value of this field does not give us any useful effect.

The results demonstrating the possibility of mechanical tuning of the isolator by changing an angle of an anisotropy axis inclination are shown in Figure 7. The minimum is shifted from $\alpha=72^{\circ}$ to $\alpha=82^{\circ}$ if an inclination angle is changed from $\beta=25^{\circ}$ to $\beta=40^{\circ}$. Simultaneously, the angle bandwidth is narrowed and it is equal to $28^{\circ}$, $10^{\circ}$, and $6^{\circ}$ correspondingly. Decreasing the inclination angle less than $\beta=25^{\circ}$ and increasing it more than $\beta=50^{\circ}$ leads to disappearance of the angle selective properties of the structure.

Figure 8 shows the results of calculations, allowing us to improve the described isolator. It is seen in Figure 8 that the reflection minimum is observed at $\alpha=76.4^{\circ}$ when the frequency is $6.35 \mathrm{GHz}$ and the reflection minimum 


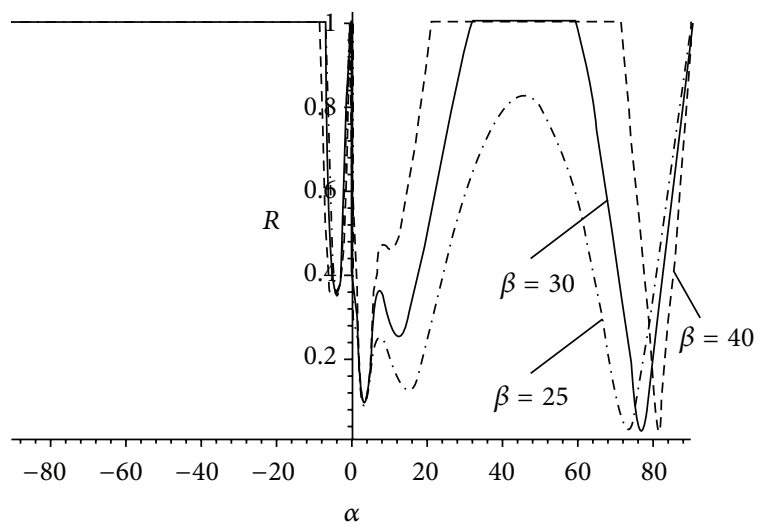

FIgURE 7: Dependence of the reflection coefficient on an incidence angle for difference inclination angles $\beta, d_{1}=0.78 \mathrm{~mm}, d_{1} / d_{2}=$ 1.3125 , and $f=6.35 \cdot 10^{12}$.

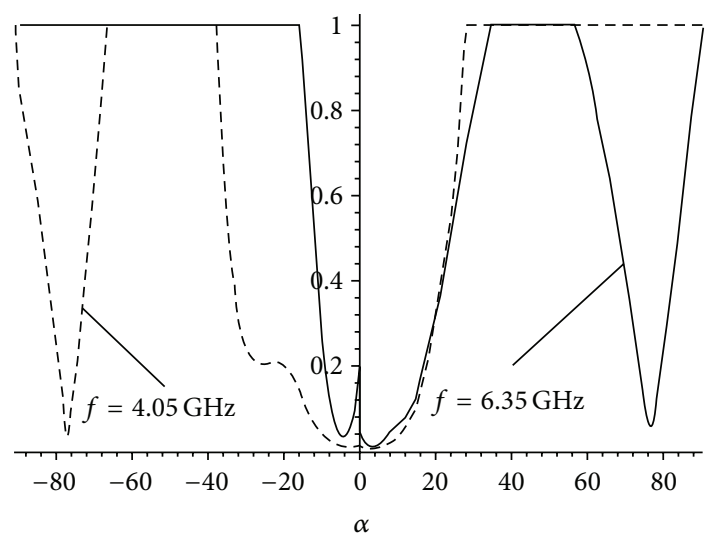

FIGURE 8: The dependence of the reflection coefficient on an incident angle for $d_{1}=0.78 \mathrm{~mm}, d_{1} / d_{2}=1.3125, \beta=30^{\circ}$; (1) $\psi=50^{\circ}$ and (2) $\psi=-50^{\circ}$.

corresponds to $\alpha=-76.4^{\circ}$ at the frequency $4.05 \mathrm{GHz}$. Thus, the structure can be used in duplex terahertz systems as a two-way isolator. Moreover, when the direction of the magnetic field is switched from $\psi=50^{\circ}$ to $\psi=-50^{\circ}$ the above minima are reversed. Therefore, if voltage is applied to solenoid 1 then a signal transmits from antenna 1 to antenna 2 substantially without attenuation at frequency $6.35 \mathrm{GHz}$, and a signal transmits from antenna 2 to antenna 1 without attenuation at frequency $4.05 \mathrm{GHz}$. If voltage is applied to solenoid 2 then a signal transmits from antenna 2 to antenna 1 substantially without attenuation at frequency $6.35 \mathrm{GHz}$, and a signal transmits from antenna 1 to antenna 2 without attenuation at a frequency $4.05 \mathrm{GHz}$.

\section{Conclusions}

Electromagnetic properties of a stratified anisotropic slab with an arbitrary orientation of the anisotropy axis are studied analytically and numerically. The translation and reflection matrices methods are used. The analytical expression of the translation matrix is obtained in the convenient compact form for the first time. The dependence of the eigenwave wavenumbers in an anisotropic slab and reflection coefficient on an anisotropy axis orientation and an incidence angle is investigated. It is shown that the structure shows nonreciprocal properties. It is important that these properties are not based on the Faraday effect. Although this effect is still inherently present in an anisotropic structure, the controlled two-way dual-frequency (duplex) isolator is presented here for the first time. Its operation principle is based on the properties of an anisotropic structure described here for the first time. The very complicated geometry of an anisotropic slab is used in this isolator. But this geometry (an arbitrary orientation of magnetic field) allows us to obtain the necessary properties of the isolator and to control a resonant frequency and an angle of a wave transmission mechanically.

\section{Appendix}

Finding a solution of Maxwell equations in the form $\exp \left(j\left(\omega t-k_{x} x-k_{y} y-k_{z} z\right)\right)$, we can write

$$
\begin{aligned}
& \left|\begin{array}{cccccc}
0 & -j k_{z} & j k_{y} & -j \omega \mu_{x x} & -j \omega \mu_{x y} & -j \omega \mu_{x z} \\
j k_{z} & 0 & -j k_{x} & -j \omega \mu_{y x} & -j \omega \mu_{y y} & -j \omega \mu_{x z} \\
-j k_{y} & j k_{x} & 0 & -j \omega \mu_{z x} & -j \omega \mu_{z y} & -j \omega \mu_{z z} \\
j \omega \varepsilon & 0 & 0 & 0 & -j k_{z} & j k_{y} \\
0 & j \omega \varepsilon & 0 & j k_{z} & 0 & -j k_{x} \\
0 & 0 & j \omega \varepsilon & -j k_{y} & j k_{x} & 0
\end{array}\right|\left|\begin{array}{c}
E_{x} \\
E_{y} \\
E_{z} \\
H_{x} \\
H_{y} \\
H_{z}
\end{array}\right| \\
& =0 .
\end{aligned}
$$

From (A.1), we have

$$
\begin{aligned}
& \left|\begin{array}{ccccc}
0 & -j k_{z} & j k_{y} & -j \omega \mu_{x x} & -j \omega \mu_{x y} \\
j k_{z} & 0 & -j k_{x} & -j \omega \mu_{y x} & -j \omega \mu_{y y} \\
-j k_{y} & j k_{x} & 0 & -j \omega \mu_{z x} & -j \omega \mu_{z y} \\
j \omega \varepsilon & 0 & 0 & 0 & -j k_{z} \\
0 & j \omega \varepsilon & 0 & j k_{z} & 0
\end{array}\right|\left|\begin{array}{c}
E_{x} \\
E_{y} \\
E_{z} \\
H_{x} \\
H_{y}
\end{array}\right| \\
& =\left|\begin{array}{c}
j \omega \mu_{x z} \\
j \omega \mu_{y z} \\
j \omega \mu_{z z} \\
-j k_{y} \\
j k_{x}
\end{array}\right| H_{z} .
\end{aligned}
$$

The solutions of (A.2) give us the expressions of the matrix elements in (5) in the form

$$
\gamma_{m i}=\frac{\Delta_{m i}}{\Delta}
$$


where $\Delta$ is the determinant of the coefficient matrix in (A.3) and

$$
\begin{aligned}
\Delta_{1 i} & =\left|\begin{array}{ccccc}
j \omega \mu_{x z} & -j k_{z i} & j k_{y} & -j \omega \mu_{x x} & -j \omega \mu_{x y} \\
j \omega \mu_{y z} & 0 & -j k_{x} & -j \omega \mu_{y x} & -j \omega \mu_{y y} \\
j \omega \mu_{z z} & j k_{x} & 0 & -j \omega \mu_{z x} & -j \omega \mu_{z y} \\
-j k_{y} & 0 & 0 & 0 & -j k_{z i} \\
j k_{x} & j \omega \varepsilon & 0 & j k_{z i} & 0
\end{array}\right|, \\
\Delta_{2 i}= & \left|\begin{array}{ccccc}
0 & j \omega \mu_{x z} & j k_{y} & -j \omega \mu_{x x} & -j \omega \mu_{x y} \\
j k_{z i} & j \omega \mu_{y z} & -j k_{x} & -j \omega \mu_{y x} & -j \omega \mu_{y y} \\
-j k_{y} & j \omega \mu_{z z} & 0 & -j \omega \mu_{z x} & -j \omega \mu_{z y} \\
j \omega \varepsilon & -j k_{y} & 0 & 0 & -j k_{z i} \\
0 & j k_{x} & 0 & j k_{z i} & 0
\end{array}\right|, \\
\Delta_{3 i}= & \left|\begin{array}{ccccc}
0 & -j k_{z i} & j k_{y} & j \omega \mu_{x z} & -j \omega \mu_{x y} \\
j k_{z i} & 0 & -j k_{x} & j \omega \mu_{y z} & -j \omega \mu_{y y} \\
-j k_{y} & j k_{x} & 0 & j \omega \mu_{z z} & -j \omega \mu_{z y} \\
j \omega \varepsilon & 0 & 0 & -j k_{y} & -j k_{z i} \\
0 & j \omega \varepsilon & 0 & j k_{x} & 0 \\
j k_{z i} & 0 & -j k_{x} & -j \omega \mu_{y x} & j \omega \mu_{y z} \\
-j k_{y} & j k_{x} & 0 & -j \omega \mu_{z x} & j \omega \mu_{z z} \\
j \omega \varepsilon & 0 & 0 & 0 & -j k_{y} \\
0 & j \omega \varepsilon & 0 & j k_{z i} & j k_{x}
\end{array}\right|,
\end{aligned}
$$

\section{Conflict of Interests}

The authors declare that there is no conflict of interests regarding the publication of this paper.

\section{References}

[1] R. J. Potton, "Reciprocity in optics," Reports on Progress in Physics, vol. 67, no. 5, pp. 717-754, 2004.

[2] A. Shuvaev, A. Pimenov, G. V. Astakhov et al., "Room temperature electrically tunable terahertz Faraday effect," Applied Physics Letters, vol. 102, no. 24, Article ID 241902, 2013.

[3] M. Shalaby, F. Vidal, M. Peccianti et al., "Terahertz macrospin dynamics in insulating ferrimagnets," Physical Review B, vol. 88, no. 14, Article ID 140301, 2013.

[4] M. Rotter, W. Ruile, and A. Wixforth, "Non-reciprocal SAW devices for RF applications," in Proceedings of the IEEE Ultrasonics Symposium 2000, vol. 1, pp. 35-38, San Juan, Puerto Rico, October 2000.

[5] J. Koch, A. A. Houck, K. L. Hur, and S. M. Girvin, “Timereversal-symmetry breaking in circuit-QED-based photon lattices," Physical Review A, vol. 82, no. 4, Article ID 043811, 2010.

[6] N. R. Anderson and R. E. Camley, "Attenuated total reflection study of bulk and surface polaritons in antiferromagnets and hexagonal ferrites: propagation at arbitrary angles," Journal of Applied Physics, vol. 113, no. 1, Article ID 013904, 2013.
[7] T. J. Fal and R. E. Camley, "Non-reciprocal devices using attenuated total reflection and thin film magnetic layered structures," Journal of Applied Physics, vol. 110, no. 5, Article ID 053912, 2011.

[8] M. Shalaby, M. Peccianti, Y. Ozturk, and R. Morandotti, "A magnetic non-reciprocal isolator for broadband terahertz operation," Nature Communications, vol. 4, article 1558, 2013.

[9] S. Chen, F. Fan, X. Wang, P. Wu, H. Zhang, and S. Chang, "Terahertz isolator based on nonreciprocal magneto-metasurface," Optics Express, vol. 23, no. 2, pp. 1015-1024, 2015.

[10] M. R. F. Jensen, T. J. Parker, K. Abraha, and D. R. Tilley, "Experimental observation of magnetic surface polaritons in $\mathrm{FeF}_{2}$ by attenuated total reflection," Physical Review Letters, vol. 75, no. 20, pp. 3756-3759, 1995.

[11] M. R. F. Jensen, S. A. Feiven, T. Dumelow et al., "Fourier transform spectroscopy of magnetic materials at terahertz frequencies," International Journal of Terahertz Science and Technology, vol. 2, no. 4, pp. 105-119, 2009.

[12] B. Lax and K. Button, Microwave Ferrites and Ferrimagnetics, McGRaw-Hill Book, 1962.

[13] P. Yeh, A. Yariv, and C. S. Hong, "Electromagnetic propagation in periodic stratified media. I. General theory," Journal of the Optical Society of America, vol. 67, no. 4, pp. 423-436, 1977.

[14] D. W. Berreman, "Optics in stratified and anisotropic media: $4 \times$ 4 matrix formulation," Journal of the Optical Society of America, vol. 62, no. 4, pp. 502-510, 1972.

[15] S. Teitler and B. W. Henvis, "Refraction in stratified anisotropic media," Journal of the Optical Society of America, vol. 60, no. 6, pp. 830-834, 1970.

[16] F. Fedorov, Optics of Anisotropic Media, Editorial URSS, 2004. 


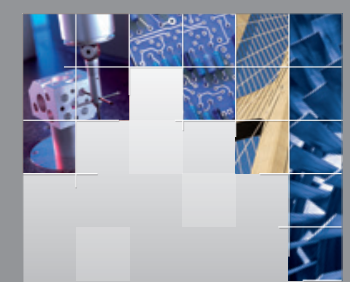

\section{Enfincering}
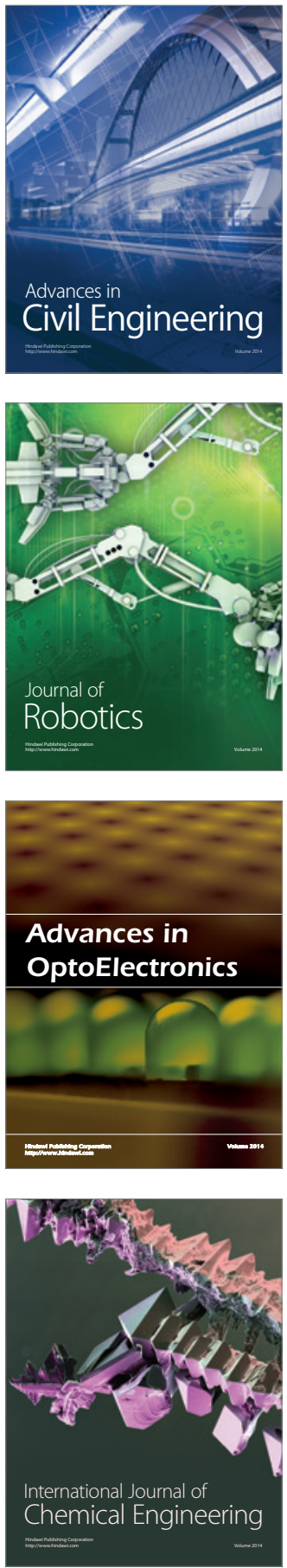

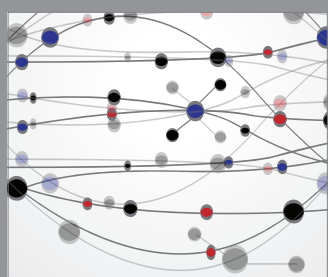

The Scientific World Journal

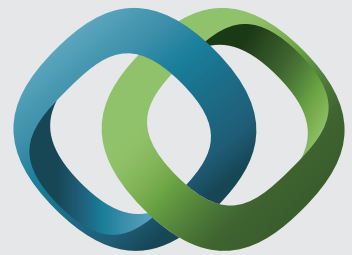

\section{Hindawi}

Submit your manuscripts at

http://www.hindawi.com
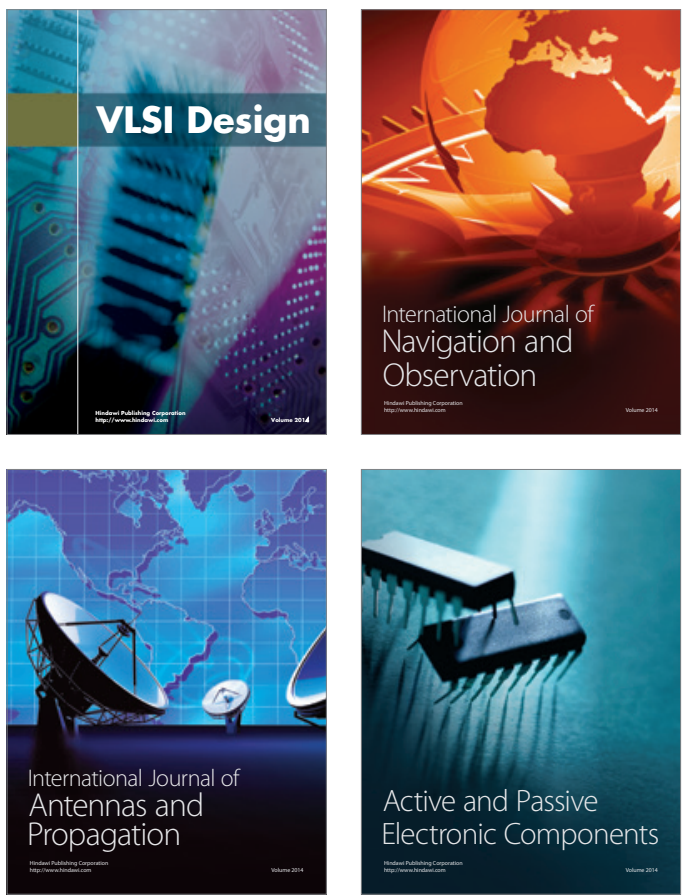
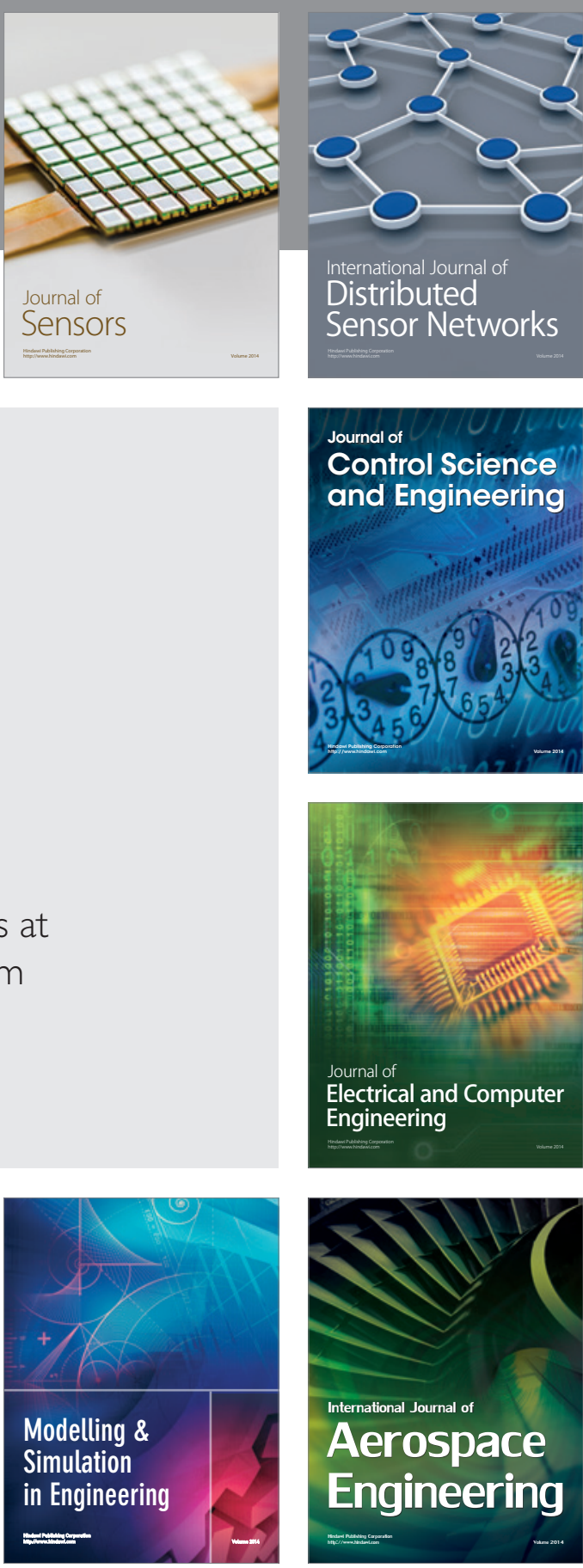

International Journal of

Distributed

Sensor Networks

Journal of

Control Science

and Engineering
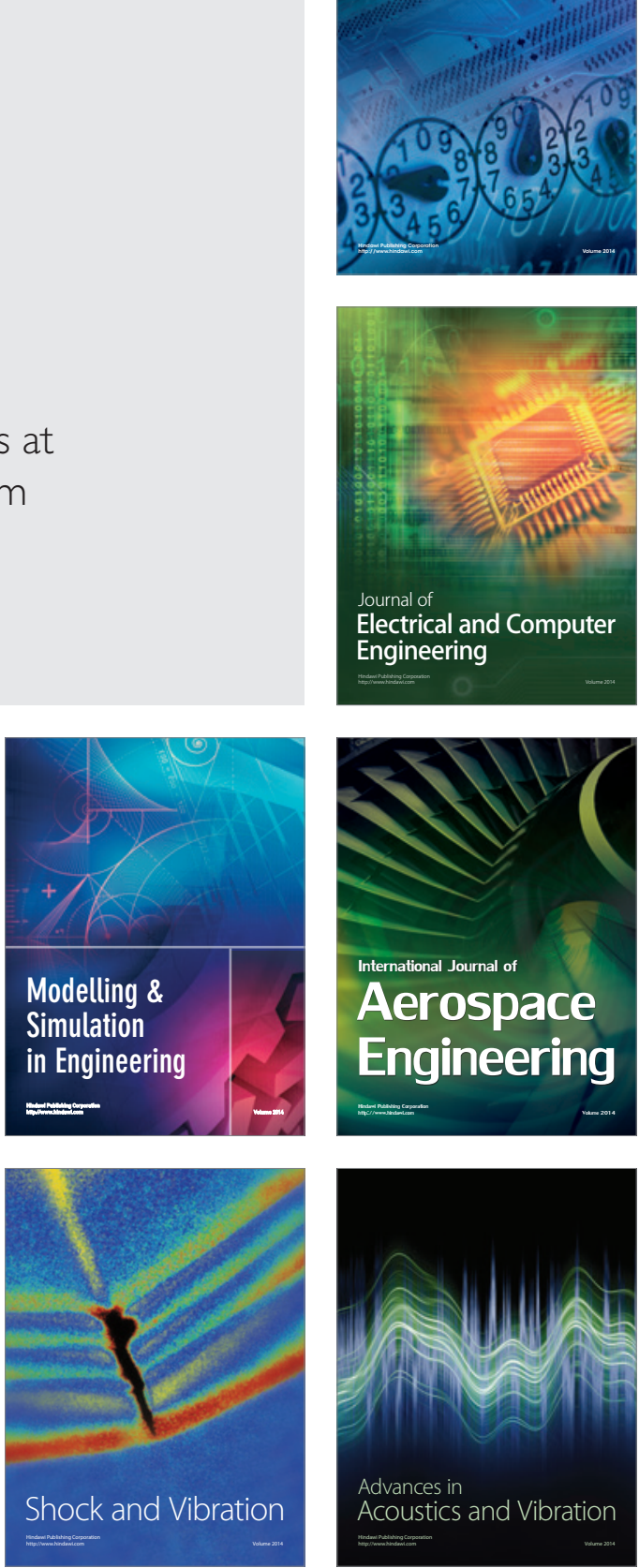